\title{
Chronic inflammatory diseases, anti- inflammatory medications and risk of prostate cancer: a population-based case- control study
}

Kerri Beckmann ${ }^{1,2^{*}}$ (D), Beth Russell ${ }^{2}$, Debra Josephs ${ }^{2}$, Hans Garmo ${ }^{2,3}$, Christel Haggstrom ${ }^{4,5}$, Lars Holmberg ${ }^{2,5}$, Pär Stattin ${ }^{5}$, Mieke Van Hemelrijck ${ }^{2,6+}$ and Jan Adolfsson ${ }^{7+}$

\begin{abstract}
Background: Whether chronic inflammation increases prostate cancer risk remains unclear. This study investigated whether chronic inflammatory diseases (CID) or anti-inflammatory medication use (AIM) were associated with prostate cancer risk.

Methods: Fifty-five thousand nine hundred thirty-seven cases (all prostate cancer, 2007-2012) and 279,618 age-matched controls were selected from the Prostate Cancer Database Sweden. CIDs and AIMs was determined from national patient and drug registers. Associations were investigated using conditional logistic regression, including for disease/drug subtypes and exposure length/dose.

Results: Men with a history of any CID had slightly increased risk of any prostate cancer diagnosis (OR: 1.08; 95\%Cl: 1.041.12) but not 'unfavourable' (high-risk or advanced) prostate cancer. Generally, risk of prostate cancer was highest for shorter exposure times. However, a positive association was observed for asthma $>5$ years before prostate cancer diagnosis (OR: 1.21; 95\%Cl: 1.05-1.40). Risk of prostate cancer was increased with prior use of any AIMs (OR: 1.26 ; $95 \% \mathrm{Cl}$ : 1.24-1.29). A positive trend with increasing cumulative dose was only observed for inhaled glucocorticoids $(p<0.011)$.

Conclusion: Detection bias most likely explains the elevated risk of prostate cancer with prior history of CIDs or use of AIMs, given the higher risk immediately after first CID event and lack of dose response. However, findings for length of time with asthma and dose of inhaled glucocorticoids suggest that asthma may increase risk of prostate cancer through other pathways.
\end{abstract}

Keywords: Prostate cancer, Chronic inflammatory disease, Autoimmune disease, Anti-inflammatory medication

\section{Background}

There is convincing evidence that some specific cancers are associated with chronic inflammatory diseases (CIDs). Examples include inflammatory bowel disease and colon cancer, Helicobacter Pylori infection and gastric cancer, hepatitis $B$ and $C$ infections and hepatocellular carcinoma [1]. Evidence for chronic inflammation as a risk factor for

\footnotetext{
* Correspondence: Kerri.beckmann@kcl.ac.uk

${ }^{+}$Mieke Van Hemelrijck Jan Adolfsson contributed equally as senior author

${ }^{1}$ UniSA Cancer Research Institute, University of South Australia, Adelaide, Australia

${ }^{2}$ School of Cancer and Pharmaceutical Studies, Translational Oncology \& Urology Research (TOUR), King's College London, London, UK

Full list of author information is available at the end of the article
}

prostate cancer is less clear. Several different lines of evidence suggest that inflammation plays a role in prostate cancer development. For example, a number of putative risk factors for prostate cancer induce prostate inflammation, including heterocyclic amines in highly cooked meat, prostatitis and sexually transmitted diseases (STDs) [2-5]. In addition to localised inflammation, there is evidence suggesting systemic inflammation may also increase prostate cancer risk. High levels of circulating C-reactive protein [6], a marker of inflammation, and increased Immunoglobulin (Ig) E levels [7], a marker of atopy, are associated with increased risk of prostate cancer. Increased risk of prostate cancer has been reported among men with asthma or other

(c) The Author(s). 2019 Open Access This article is distributed under the terms of the Creative Commons Attribution 4.0 International License (http://creativecommons.org/licenses/by/4.0/), which permits unrestricted use, distribution, and reproduction in any medium, provided you give appropriate credit to the original author(s) and the source, provide a link to the Creative Commons license, and indicate if changes were made. The Creative Commons Public Domain Dedication waiver (http://creativecommons.org/publicdomain/zero/1.0/) applies to the data made available in this article, unless otherwise stated. 
atopic conditions $[7,8]$ and among men with autoimmune diseases [9-12], though contradictory findings have also been reported [13-17]. On the other hand, reduced risk of prostate cancer has been reported with exposure to some anti-inflammatory medications (AIMs) used to manage CIDs, though again the evidence is mixed [18-21].

However, previous evidence of positive associations between CIDs/AIMs and prostate cancer may be affected by detection bias, resulting from frequent health service encounters or investigations leading to detection of prostate cancer, especially for men with localised inflammation arising from prostatitis or STDs [22].

The aim of this study was to investigate the link between chronic inflammation and risk of prostate cancer by examining the association between 1 ) history of CIDs, and 2) use of AIMs, and prostate cancer incidence, using population-wide registry-based data. Our a priori hypotheses were that risk of prostate cancer would be increased among men with CIDs due to persistent or recurring exposure to inflammatory mediators, while risk would be reduced among men who used AIMs, due to anti-inflammatory properties of these drugs.

\section{Methods}

\section{Data source and variables}

We used data from Prostate Cancer Database Sweden (PCBaSe), a population-wide database linkage between the National Prostate Cancer Registry (NPCR), the National Patient Register (NPR), the National Prescribed Drug Register (from Jan 1, 2006) and the Swedish longitudinal integration database for health insurance and labour market studies (LISA), through Swedish personal identification numbers [23]. In addition to men diagnosed with prostate cancer, PCBaSe also includes disease-free population controls, selected using an incident density sampling approach [23]. The NPCR has near complete coverage (98\%) of prostate cancer diagnoses across Sweden since 1988. Information collected in NPCR includes date of diagnosis, age, tumour stage and grade, levels of prostate specific antigen (PSA) at diagnosis, and primary treatment.

We used a case-control design to study the association between CIDs/AIMs and risk of prostate cancer diagnosis. The main outcome of this study was a diagnosis of prostate cancer, recorded in NPCR. All men diagnosed with prostate cancer between Jan 1, 2007 and Dec 31, 2012 were selected as cases. Five population controls, free of prostate cancer at the time of diagnosis of the corresponding index case, matched on year of birth and area of residence, were selected for each case.

The two broad exposures of interest were 1) prior history of CIDs and 2) exposure to AIMs. For the CIDs component, the analytic study population was restricted to men diagnosed from 2007 to 2009 plus controls, due to incomplete data on rare conditions from 2010 onwards. Prior history of CIDs was derived from International classification of diseases, 10th edition (ICD-10) codes recorded in the NPR for any single hospital inpatient or outpatient episode from 1998 onwards. CIDs were classified into the following subcategories: chronic inflammatory and infectious diseases; allergies; and autoimmune diseases (further subgrouped as auto-antibody negative; auto-antibody positive - systemic; and auto-antibody positive - organ specific diseases). Type 1 diabetes mellitus (T1DM) was excluded from the organ specific autoimmune category given likely misclassification of type 2 diabetes mellitus. Likewise, prostatitis was excluded from the chronic inflammatory diseases category due to likely detection bias from more intensive investigation of the prostate. Both were analysed separately. The full list of included diseases and respective ICD-10 codes is provided in the Additional file 2.

For analysis of AIMs, the whole study population was included. Exposure to selected AIMs was determined through linkage to Sweden's National Prescribed Drug register, based on Anatomical Therapeutic Chemical (ATC) codes for drugs commonly prescribed for the management of CIDs: Non-aspirin non-steroidal anti-inflammatory drugs (NSAID) (M01A), systemic glucocorticoids (H02AB), inhaled glucocorticoids (R03BA), non-steroidal asthma medications (R03AC, R03CC, R03AK, R03BB, R03DC), immunosuppressants (L04A), and anti-gout medications (M04A). One previous prescription was considered to be sufficient to be classified as exposed. Data were extracted on class of drug, date of first prescription and cumulative dose before diagnosis. These data were only available from July 1, 2005, onwards. For both CIDs and AIMs, length of exposure and cumulative dose were determined for similar periods for cases and controls, with date of diagnosis of the case being used as the reference date for selected controls.

Charlson comorbidity index [24] (CCI) was derived from data hospital admission records prior to diagnosis of prostate cancer. Marital status and education level were extracted from LISA, while data on prostate cancer risk categories (grade, clinical stage, and prostate serum antigen (PSA) level at diagnosis) were extracted from NPCR. To determine whether CIDs or AIMs were associated with disease severity at diagnosis we classified men with Prostate cancer according to risk categories. The 'favourable risk prostate cancer' group included low and intermediate risk categories (Gleason Score $<=7$, clinical stage $\mathrm{T} 1-2, \mathrm{PSA} \leq 20, \mathrm{~N} 0 / \mathrm{X}$ and $\mathrm{M} 0 / \mathrm{X}]$ ), while the 'unfavourable risk' prostate cancer group included high-risk, regionally-advanced and metastatic disease (Gleason score $=8-10$, PSA $>20, \mathrm{~N} 1$ or M1). These terms are used below to refer to different risk groupings.

\section{Statistical analysis}

Associations between CIDs/AIMs and prostate cancer risk were examined using multivariable conditional logistic 
regression. Separate analyses were undertaken for any prior CIDs and any AIMs exposure, as well as each CID and AIM subtype. In all cases the reference group were all men not previously exposed to the specific condition or medication of interest. To assess the potential for detection bias, we examined length of time since first recorded diagnosis of CID $(<12,12-<36,36-<60,60+$ months $)$ and total cumulative dose for subtypes of AIMs ( $<50,50-<200,200-<$ $500,500+$ daily dose equivalents) prior to prostate cancer diagnosis. Models were adjusted for education level (low< 10 years, middle $10-12$ years, high $>12$ years); marital status (married, not married), and comorbidity $(\mathrm{CCI}=0,1,2,3+$ ). Wald tests were used to test trends according to categories of exposure for CIDs and cumulative daily dose for AIMs. Separate subgroup analyses were also undertaken restricted to 'favourable' and 'unfavourable' risk groups and their respective controls.

We also investigated interaction between history of CIDs and major classes of anti-inflammatory medications. For these analyses, interaction terms for CIDs and AIMs subtype were included in regression models for overall risk of prostate cancer, along with other covariates. Differences in log likelihood between models with and without interaction terms were used to determine any statistically significant effect modification in men with CID history.

Finally we undertook a series of sensitivity analyse to examine the robustness of our findings with respect to AIMs and prostate cancer risk. These included 1) altering our definition of AIMs exposure to 2 or more prior prescriptions, 2) restricting the cohort to men diagnosed with prostate cancer from 2010 to 2012 to allow for longer time for AIMs exposure given prescription data were only available from 2006, and 3) excluding cases whose prostate cancer diagnosis was within 12 months of their first prescription.

All statistical analyses were undertaken using Stata v 14.0 (Stata Corporation, College Station, Texas USA). This study was approved by the Research Ethics Board, Umeå University, Sweden.

\section{Results}

The study population included 55,937 men with prostate cancer diagnosed between 2007 and 2012 and 279,618 controls. Among cases, 32,193 (57\%) had favourable risk prostate cancer, 22,275 (43\%) had unfavourable risk prostate cancer, and 1469 (3\%) had unknown risk category. Table 1 presents demographic characteristics of the study population. Mean age at diagnosis and selection as controls was 69 years (standard deviation $=9$ ). The proportion of men with a prior record of CIDs was similar between cases and controls (20\% vs $19 \%)$. The proportion of men previously prescribed AIMs (all medications combined) was slightly higher for cases than controls (56\% vs $50 \%)$.

Chronic inflammatory conditions and prostate cancer risk Adjusted odds ratios (ORs) for associations between history of CIDs and risk of prostate cancer are shown in Table 2. Results indicate a modest positive association between history of any CIDs and risk of prostate cancer (OR: 1.08; 95\% CI 1.04-1.12). Positive associations were also observed for two disease sub-categories: chronic inflammatory/infectious diseases (OR: 1.07: 95\%CI: 1.031.12 ) and allergies (OR: 1.10; 95\%CI 1.03-1.18) and specifically for asthma within the allergies category (OR: 1.13, $95 \%$ CI 1.02-1.25). No association was observed for autoimmune diseases, including by antibody status or whether systemic or organ confined. We found moderately decreased risk of prostate cancer among men with T1DM (OR: 0.71; 95\%CI: 0.65-0.78) and elevated risk among men with chronic prostatitis (OR: 1.82; 95\%CI: 1.53-2.16).

Associations between CIDs and prostate cancer risk groups are also shown in Table 2. For all CIDs combined, and for the inflammatory/infectious diseases and allergies subcategories, risk was only increased for favourable risk prostate cancer. Only history of chronic

Table 1 Characteristics of the study population

\begin{tabular}{lll}
\hline Cohort Characteristics & Cases & Controls \\
& $n=55,937$ & $n=279,618$ \\
\hline Age at diagnosis (years) & No. $(\%)$ & No. $(\%)$ \\
$<50$ & $526(1)$ & $2656(1)$ \\
$50-59$ & $6716(12)$ & $33,583(12)$ \\
$60-69$ & $22,532(40)$ & $112,544(40)$ \\
$70-79$ & $17,582(31)$ & $87,963(31)$ \\
$80+$ & $8581(15)$ & $42,872(15)$ \\
Marital status & & \\
Single/divorced/widowed & $18,753(34)$ & $103,830(37)$ \\
Married & $37,184(66)$ & $175,788(63)$ \\
Education level & & \\
$<9 y r s$ & $20,447(37)$ & $107,490(38)$ \\
$10-12$ yrs & $21,532(38)$ & $106,261(38)$ \\
$>12$ yrs & $13,532(24)$ & $61,699(22)$ \\
missing & $447(1)$ & $4168(1)$ \\
Charlson Comorbidity Index & & $139,311(50)$ \\
0 & $15,268(66)$ & $73,485(64)$ \\
1 & $3722(17)$ & $19,636(17)$ \\
2 & $2369(10)$ & $11,618(10)$ \\
3+ & $1824(7)$ & $11,169(9)$ \\
Period of diagnosis/inclusion & & \\
$2007-2009$ & $27,874(50)$ & $140,307(50)$ \\
\hline
\end{tabular}


Table 2 Prior history of chronic inflammatory disease (CID) and odds of prostate cancer, overall and by disease category ${ }^{a}$

\begin{tabular}{|c|c|c|c|c|c|}
\hline \multirow[t]{2}{*}{ Prior record of chronic inflammatory disease ${ }^{b}$} & \multirow{2}{*}{$\begin{array}{l}\text { Controls } n= \\
139,311 \\
\text { No. (\%) }\end{array}$} & \multirow{2}{*}{$\begin{array}{l}\text { Cases } n= \\
27,874 \\
\text { No. (\%) }\end{array}$} & \multirow{2}{*}{$\begin{array}{l}\text { Overall PCa } \\
\text { OR }(95 \% \text { Cl) }\end{array}$} & \multirow{2}{*}{$\begin{array}{l}\text { Favourable risk } \\
\text { PCa } \\
\text { ORC }(95 \% \text { Cl) }\end{array}$} & \multirow{2}{*}{$\begin{array}{l}\text { Unfavourable risk } \\
\text { PCa } \\
O R^{C}(95 \% \text { Cl) }\end{array}$} \\
\hline & & & & & \\
\hline $\begin{array}{l}\text { Any chronic inflammatory /autoimmune disease (excl. } \\
\text { Prostatitis and T1DM) }\end{array}$ & $26,272(19)$ & $5460(20)$ & $\begin{array}{l}1.08(1.04- \\
1.12)\end{array}$ & $1.13(1.08-1.19)$ & $1.02(0.96-1.07)$ \\
\hline Chronic inflammatory / infectious diseases (excl. Prostatitis) & $17,027(12)$ & $3520(13)$ & $\begin{array}{l}1.07(1.03- \\
1.12)\end{array}$ & $1.10(1.04-1.16)$ & $1.05(0.99-1.11)$ \\
\hline Chronic prostatitis & $492(0.4)$ & $180(0.7)$ & $\begin{array}{l}1.82(1.53- \\
2.16)\end{array}$ & $2.03(1.63-2.52)$ & $1.56(1.18-2.07)$ \\
\hline Allergies (including Asthma) & $5064(4)$ & $1084(4)$ & $\begin{array}{l}1.10(1.03- \\
1.18)\end{array}$ & $1.22(1.12-1.33)$ & $0.97(0.87-1.07)$ \\
\hline Asthma alone & $2116(2)$ & $456(2)$ & $\begin{array}{l}1.13(1.02- \\
1.25)\end{array}$ & $1.22(1.07-1.41)$ & $1.03(0.88-1.20)$ \\
\hline Autoimmune diseases (any excluding T1DM): & $7692(5)$ & $1542(6)$ & $\begin{array}{l}1.03(0.97- \\
1.09)\end{array}$ & $1.07(0.99-1.15)$ & $0.98(0.90-1.07)$ \\
\hline Autoantibody negative & $5349(4)$ & $1103(4)$ & $\begin{array}{l}1.05(0.98- \\
1.12)\end{array}$ & $1.08(0.99-1.18)$ & $1.00(0.90-1.10)$ \\
\hline Autoantibody positive - systemic & $1464(1)$ & $296(1)$ & $\begin{array}{l}1.07(0.94- \\
1.21)\end{array}$ & $1.07(0.90-1.28)$ & $1.06(0.89=1.27)$ \\
\hline Autoantibody positive - organ specific & $5221(4)$ & $774(3)$ & $\begin{array}{l}0.77(0.71- \\
0.83)\end{array}$ & $0.74(0.67-0.83)$ & $0.82(0.73-0.91)$ \\
\hline Autoantibody positive - organ specific (excl. T1DM) & $1152(1)$ & $220(1)$ & $\begin{array}{l}0.98(0.84- \\
1.12)\end{array}$ & $1.08(0.89-1.31)$ & $0.85(0.68-1.06)$ \\
\hline T1DM & $4136(3)$ & $506(2)$ & $\begin{array}{l}0.71(0.65- \\
0.78)\end{array}$ & $0.64(0.56-0.73)$ & $0.80(0.71-0.91)$ \\
\hline
\end{tabular}

Aberrations: $\mathrm{Cl}$ confidence interval, OR Odds Ratio, $P C a$ prostate cancer, T1DM Type 1 diabetes mellitus

${ }^{a}$ Favourable risk PCa: low and intermediate risk (Gleason Score $\leq 7, \mathrm{~T} 1-\mathrm{T} 2, \mathrm{PSA} \leq 20 \mathrm{ng} / \mathrm{mL}, \mathrm{N} 0 / \mathrm{X}, \& \mathrm{M0} / \mathrm{X}$ ), Unfavourable risk PCa: (Gleason score $8-10$, or PSA $>20$ $\mathrm{ng} / \mathrm{ml}$, or $\mathrm{N} 1$, or $\mathrm{M} 1$ )

${ }^{b}$ Derived from ICD-10 diagnosis codes recorded in hospital admission or outpatient records since 1997. Reference category= no record of inpatient or outpatient hospital episode

'Adjusted OR from conditional logistic regression with adjustment for civil status, education level and Charlson Comorbidity Index. Analyses stratified low/ intermediate risk and advanced disease at diagnosis along with respective matched controls. Analysis undertaken on subset diagnosed 2007-09, with complete hospital admission data from Jan 1, 1997 - Dec 31, 2009

prostatitis was associated with both favourable (OR: 2.03; 95\% CI: $1.63-2.52$ ) and unfavourable risk prostate cancer (OR: 1.56; 95\%CI: 1.18-2.07), with the former having the stronger association. The negative association observed for T1DM was evident for both favourable (OR: 0.64; 95\%CI: 0.56-0.80) and unfavourable risk prostate cancer (OR: 0.80; 95\%CI: 0.71-0.91), with a stronger association for favourable risk prostate cancer.

Table 3 shows the adjusted ORs for CIDs and risk of prostate cancer for time since first recorded hospital episode. There were no consistent trends with length of time since first diagnosis for any CIDs or specific subtypes. For prostatitis, there was a significant decreasing trend in prostate cancer risk with increasing length of exposure $(p=0.004)$. For the allergies subcategory, ORs were highest among men with their first hospital episode within 12 months of diagnosis (OR: 1.25; 95\%CI: 1.00-1.56), but were also elevated for intervals of more than 60 months (OR: 1.19; 95\%CI: 1.05-1.28). The same pattern, though more pronounced, was seen for asthma alone.
Anti-inflammatory medications and prostate cancer risk A moderate positive association was observed for exposure to any of the listed AIMs (OR: 1.26; 95\%CI: $1.24-$ 1.29) Table 4. Increased risk was observed for separate classes of AIMs including non-aspirin-NSAIDs (OR: 1.25; 95\%CI: 1.23-1.27); systemic glucocorticoids (OR: 1.14; 95\%CI: 1.11-1.17), inhaled glucocorticoids (OR: 1.12; 95\%CI: 1.08-1.17) and other non-steroidal asthma medications (OR: 1.11; 95\%CI: 1.07-1.14). Of the nonaspirin-NSAIDs, risk of prostate cancer were elevated for acetic acid derivatives (OR: 1.24; 95\%CI: 1.21-1.26), propionic acid derivatives (OR: 1.15; 95\%CI: 1.12-1.18) and Cox 2 inhibitors (OR: 1.18; 95\%CI: 1.12-1.21) and both short acting (OR: 1.09; 95\%CI: 1.03-1.16) and long acting beta agonist (OR: 1.12; 95\%CI: 1.08-1.16) nonsteroidal asthma medications. Sensitivity analyses with a stricter definition of exposure ( $\geq 2$ prescriptions), with exclusion of cases diagnosed within 12 months of their first prescription and for the diagnosis period 2010-12 did not alter the strength or direction of association for any AIMs subtypes and overall prostate cancer risk (Additional file 1: Table S1). 
Table 3 Time interval from first recorded episode for specific inflammatory disease groups and odds of prostate cancer diagnosis

\begin{tabular}{llllll}
\hline $\begin{array}{l}\text { Time from first hospital } \\
\text { episode to diagnosis/ }\end{array}$ & $<12$ & $12-<$ & $\geq 36$ & $>60$ & \\
$\begin{array}{l}\text { inclusion date } \\
\text { months }\end{array}$ & 36 & months & months & \\
& months & & & \\
& $\mathrm{OR}^{\mathrm{a}}$ & $\mathrm{OR}$ & $\mathrm{OR}$ & $\mathrm{OR}$ & p-trend $^{\mathrm{b}}$ \\
& $(95 \%$ & $(95 \%$ & $(95 \%$ & $(95 \%$ & \\
& $\mathrm{Cl})$ & $\mathrm{Cl})$ & $\mathrm{Cl})$ & $\mathrm{Cl})$ & \\
\hline
\end{tabular}

DISEASE GROUPS

(reference: no

recorded episode)

\begin{tabular}{|c|c|c|c|c|c|}
\hline $\begin{array}{l}\text { Any chronic } \\
\text { inflammatory } \\
\text { /autoimmune disease } \\
\text { (excl. Prostatitis and } \\
\text { T1DM) }\end{array}$ & $\begin{array}{l}1.01 \\
(0.90- \\
1.14)\end{array}$ & $\begin{array}{l}1.09 \\
(1.02- \\
1.16)\end{array}$ & $\begin{array}{l}1.04 \\
(0.98- \\
1.11)\end{array}$ & $\begin{array}{l}1.10 \\
(1.05- \\
1.15)\end{array}$ & 0.371 \\
\hline $\begin{array}{l}\text { Chronic inflammatory/ } \\
\text { infectious diseases (excl. } \\
\text { prostatitis) }\end{array}$ & $\begin{array}{l}0.93 \\
(0.80- \\
1.07)\end{array}$ & $\begin{array}{l}1.11 \\
(1.03- \\
1.20)\end{array}$ & $\begin{array}{l}1.07 \\
(1.03- \\
1.12)\end{array}$ & $\begin{array}{l}1.10 \\
(1.04- \\
1.16)\end{array}$ & 0.390 \\
\hline Chronic Prostatitis & $\begin{array}{l}3.93 \\
(2.15- \\
7.19)\end{array}$ & $\begin{array}{l}2.29 \\
(1.66- \\
3.16)\end{array}$ & $\begin{array}{l}1.52 \\
(1.23- \\
1.89)\end{array}$ & $\begin{array}{l}1.53 \\
(1.15- \\
2.03)\end{array}$ & 0.004 \\
\hline $\begin{array}{l}\text { Allergies (including } \\
\text { asthma) }\end{array}$ & $\begin{array}{l}1.25 \\
(1.00- \\
1.56)\end{array}$ & $\begin{array}{l}1.01 \\
(0.88- \\
1.15)\end{array}$ & $\begin{array}{l}1.04 \\
(0.91- \\
1.19)\end{array}$ & $\begin{array}{l}1.19 \\
(1.05- \\
1.28)\end{array}$ & 0.480 \\
\hline Asthma alone & $\begin{array}{l}1.63 \\
(1.14- \\
2.31)\end{array}$ & $\begin{array}{l}0.94 \\
(0.74- \\
1.18)\end{array}$ & $\begin{array}{l}0.99 \\
(0.79- \\
1.23)\end{array}$ & $\begin{array}{l}1.21 \\
(1.05- \\
1.40)\end{array}$ & 0.700 \\
\hline $\begin{array}{l}\text { Auto-immune diseases } \\
\text { (excluding T1DM) }\end{array}$ & $\begin{array}{l}1.05 \\
(0.84- \\
1.32)\end{array}$ & $\begin{array}{l}0.96 \\
(0.85- \\
1.09)\end{array}$ & $\begin{array}{l}1.03 \\
(0.91- \\
1.16)\end{array}$ & $\begin{array}{l}1.05 \\
(0.97- \\
1.13)\end{array}$ & 0.425 \\
\hline Antibody negative & $\begin{array}{l}1.13 \\
(0.87- \\
1.46)\end{array}$ & $\begin{array}{l}0.98 \\
(0.85- \\
1.13)\end{array}$ & $\begin{array}{l}1.12 \\
(0.98- \\
1.28)\end{array}$ & $\begin{array}{l}1.03 \\
(0.94- \\
1.14)\end{array}$ & 0.981 \\
\hline $\begin{array}{l}\text { Antibody positive - } \\
\text { systemic }\end{array}$ & $\begin{array}{l}0.92 \\
(0.53- \\
1.59)\end{array}$ & $\begin{array}{l}1.01 \\
(0.76- \\
1.34))\end{array}$ & $\begin{array}{l}0.99 \\
(0.74- \\
1.33)\end{array}$ & $\begin{array}{l}1.13 \\
(0.96- \\
1.33)\end{array}$ & 0.303 \\
\hline $\begin{array}{l}\text { Antibody positive - } \\
\text { organ confined (excl. } \\
\text { T1DM) }\end{array}$ & $\begin{array}{l}0.90 \\
(0.47- \\
1.69)\end{array}$ & $\begin{array}{l}1.01 \\
(0.74- \\
1.37)\end{array}$ & $\begin{array}{l}0.70 \\
(0.48- \\
1.03)\end{array}$ & $\begin{array}{l}1.06 \\
(0.87- \\
1.27)\end{array}$ & 0.474 \\
\hline T1DM only & $\begin{array}{l}0.97 \\
(0.68- \\
1.40)\end{array}$ & $\begin{array}{l}0.66 \\
(0.53- \\
0.81)\end{array}$ & $\begin{array}{l}0.71 \\
(0.64- \\
0.79)\end{array}$ & $\begin{array}{l}0.71 \\
(0.63- \\
0.81)\end{array}$ & 0.673 \\
\hline
\end{tabular}

Aberrations: $\mathrm{Cl}$ confidence interval, OR Odds Ratio, $P C a$ prostate cancer, T1DM Type 1 diabetes mellitus

${ }^{a}$ Adjusted OR from conditional logistic regression with adjustment for marital status, education level and Charlson Comorbidity Index. Analysis undertaken on subset diagnosed 2007-09, with complete hospital admission data from Jan 1, 1997 - Dec 31, 2009

${ }^{\mathrm{b}} P$-value for trend across time categories (Wald statistic)

With respect to disease severity, exposure to any AIMs was positively associated with both favourable (OR: 1.34; 95\%CI: $1.31-1.38)$ and unfavourable risk prostate cancer (OR: 1.17; 95\%CI: 1.13-1.20) but associations were stronger for the favourable risk group. Similar patterns was observed for most AIMs subtypes.

As shown in Table 5, significant trends indicating stronger associations with increasing cumulative dose were seen for inhaled glucocorticoids $(p=0.011)$ and immuno-suppressants $(p=0.001)$. However, for immune-suppressants, lower cumulative doses $(<=200$ daily dose equivalents) were associated with reduced risk of prostate cancer diagnosis, with no association at higher doses (>200 daily dose equivalents). For acetic acid derivatives there was a significant trend toward decreasing association with increasing cumulative dose $(p=0.002)$. There were no significant trends for other classes of non-aspirin-NSAIDs.

With respect to interaction between CIDs and AIMs, we observed a slight decrease in the strength of association between prior non-aspirin-NSAID use and overall prostate cancer risk among men with a history of CIDs $(P<0.04)$. Similar interactions were observed for acetic acid derivative $(p=0.007)$ and for systemic glucocorticoid exposure $(p=0.001)$ but again the effects was only slightly weaker in men with history of CIDs (Additional file 1: Table S2).

\section{Discussion}

This comprehensive, population-wide observational study found no convincing evidence of increased risk of prostate cancer among men with a history of any CIDs. While the odds ratio for any prior CID was slightly elevated (OR = 1.08), this only applied to favourable risk disease, with no elevated risk for less favourable risk prostate cancer. For specific CIDs, our results suggest increased risk of prostate cancer for chronic prostatitis and for allergies, but decreased risk among those hospitalised with T1DM. Our analyses of AIMs revealed moderately increased risk of prostate cancer with any use of AIMs, and specifically for non-aspirin-NSAID, glucocorticoids and other nonsteroidal asthma medications.

Detection bias most likely explains the association between prostate cancer and history of any CID or use of AIMs, given more frequent contact with the healthcare system would increase the opportunity for prostate cancer detection through blood tests which include PSA measurement. As in most other countries, routine PSA screening is not recommended in Sweden [25]. Nevertheless, opportunistic screening occurs frequently in Sweden, with a 5 -year prevalence estimated at $60 \%$ in 2010 [26, 27]. Physicians attitudes and recommendations influence uptake of PSA testing [28, 29] and PSA testing frequency appears to be increased among those in regular contact with the healthcare system [30].

While several previous studies have reported associations between prostatitis and prostate cancer, debate is ongoing regarding whether the association is causal -i.e. via inflammatory pathways - or due to detection bias given more prostate-specific examinations [2]. Our results support the role of detection bias, since risk was highest for chronic prostatitis admissions within 12 months of diagnosis. However, given the elevated odds for chronic prostatitis $>60$ 
Table 4 Prior exposure to anti-inflammatory medications and the odds of prostate cancer, overall and by disease category ${ }^{\mathrm{a}}$

\begin{tabular}{|c|c|c|c|c|c|}
\hline \multirow[t]{2}{*}{$\begin{array}{l}\text { Exposure anti-inflammatory } \\
\text { medications }{ }^{b}\end{array}$} & Controls $n=279,618$ & Cases $n=55,937$ & Overall PCa & $\begin{array}{l}\text { Favourable risk } \\
\text { PCa }\end{array}$ & $\begin{array}{l}\text { Unfavourable risk } \\
\text { PCa }\end{array}$ \\
\hline & No. \% & No. \% & $\mathrm{OR}^{\mathrm{c}}(95 \% \mathrm{Cl})$ & $\mathrm{OR}^{\mathrm{C}}(95 \% \mathrm{Cl})$ & $\mathrm{OR}^{\mathrm{C}}(95 \% \mathrm{Cl})$ \\
\hline Any Anti-inflammatory medication & $141,172(50)$ & $31,330(56)$ & $1.26(1.24-1.29)$ & $1.34(1.31-1.38)$ & $1.17(1.13-1.20)$ \\
\hline Any Non-Aspirin-NSAIDs & $112,742(40)$ & $25,591(46)$ & $1.25(1.23-1.27)$ & $1.33(1.30-1.36)$ & $1.15(1.12-1.19)$ \\
\hline Acetic acid derivatives & $79,279(28)$ & $18,332(33)$ & $1.24(1.21-1.26)$ & $1.29(1.26-1.33)$ & $1.16(1.12-1.20)$ \\
\hline Propionic acid derivatives & $43,766(16)$ & $9817(18)$ & $1.15(1.12-1.18)$ & $1.22(1.18-1.26)$ & $1.07(1.03-1.11)$ \\
\hline Cox inhibitors & $7741(3)$ & $1828(3)$ & $1.18(1.12-1.25)$ & $1.24(1.16-132)$ & $1.10(1.01-1.20)$ \\
\hline Oxicams & $2085(1)$ & $456(1)$ & $1.09(0.98-1.21)$ & $1.24(1.09-1.41)$ & $0.87(0.73-1.04)$ \\
\hline Other non-Aspirin NSAIDs & $15,093(5)$ & $3334(6)$ & $1.10(1.06-1.14)$ & $1.25(1.19-1.32)$ & $0.92(0.87-0.98)$ \\
\hline Any non-topical glucocorticoid & $43,052(15)$ & $9500(17)$ & $1.14(1.11-1.17)$ & $1.19(1.15-1.23)$ & $1.08(1.04-1.12)$ \\
\hline Systemic glucocorticoids & $33,616(12)$ & 7449 (13) & $1.14(1.11-1.18)$ & $1.20(1.16-1.25)$ & $1.08(1.03-1.12)$ \\
\hline Inhaled glucocorticoids & $15,111(5)$ & $3343(6)$ & $1.12(1.08-1.17)$ & $1.15(1.09-1.21)$ & $1.09(1.03-1.15)$ \\
\hline Any (nonsteroidal) asthma medications & $23,205(8)$ & $4948(9)$ & $1.11(1.07-1.14)$ & $1.12(1.07-1.17)$ & $1.09(1.03-1.14)$ \\
\hline Short term b-agonists & $6171(2)$ & $1302(2)$ & $1.09(1.03-1.16)$ & $1.10(1.02-1.20)$ & $1.07(0.98-1.17)$ \\
\hline Long term b-agonists & $17,840(6)$ & $3850(7)$ & $1.12(1.08-1.16)$ & $1.11(1.06-1.17)$ & $1.12(1.06-1.18)$ \\
\hline LAMA & $7202(3)$ & $1441(3)$ & $1.06(1.00-1.12)$ & $1.06(0.98-1.15)$ & $1.05(0.97-1.14)$ \\
\hline LTRAs & $1579(1)$ & $340(1)$ & $1.08(0.96-1.21)$ & $1.10(0.95-1.28)$ & $1.05(0.87-1.27)$ \\
\hline Anti-Gout medications & $12,910(5)$ & $2568(5)$ & $1.03(0.98-1.07)$ & $1.00(0.94-1.07)$ & $1.05(0.99-1.12)$ \\
\hline Immuno-suppressants & $4281(2)$ & $812(1)$ & $0.98(0.91-1.06)$ & $0.98(0.88-1.08)$ & $1.00(0.89-1.13)$ \\
\hline
\end{tabular}

Cl confidence interval, LAMA long acting muscarinic antagonists, LTRAs leukotriene receptor antagonists, NSAIDs non-steroidal anti-inflammatory drugs, OR: odds ratio

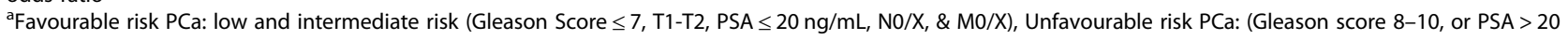
$\mathrm{ng} / \mathrm{ml}$, or $\mathrm{N} 1$, or $\mathrm{M} 1$ )

${ }^{b}$ Any exposure to anti-inflammatory medications since July 2005, derived from the National Prescribed Drug Register based on anatomical therapeutic chemical (ATC) codes

${ }^{\mathrm{C}}$ Adjusted OR from conditional logistic regression with adjustment for marital status, education level and Charlson comorbidity index. Analysis undertaken in the full dataset (2007-2012)

months prior to prostate cancer diagnosis, it is possible that other pathways may be involved, though this is difficult to disentangle from ongoing prostatic investigations.

Previous studies have consistently reported lower risk of prostate cancer in men with diabetes [31-34]. Proposed explanations include decreased hormone levels which may slow prostate carcinogenesis, potential effects of diabetes or anti-diabetic medications on serum PSA levels or prostate tissue pathology which lead to delays in diagnosis, and reduced screening/health seeking behaviour among

Table 5 Cumulative dose for selected classes of anti-inflammatory medications and risk of prostate cancer

\begin{tabular}{llllll}
\hline $\begin{array}{l}\text { Cumulative dose of anti-inflammatory } \\
\text { medication }^{\mathrm{a}}\end{array}$ & $\begin{array}{l}<=50 \mathrm{dd} \\
\mathrm{OR}(95 \% \mathrm{Cl})\end{array}$ & $\begin{array}{l}51-200 \mathrm{dd} \\
\mathrm{OR}^{\mathrm{b}}(95 \% \mathrm{Cl})\end{array}$ & $\begin{array}{l}201-500 \mathrm{dd} \\
\mathrm{OR}^{\mathrm{b}}(95 \% \mathrm{Cl})\end{array}$ & $\begin{array}{l}>500 \mathrm{dd} \\
\mathrm{OR}^{\mathrm{b}}(95 \% \mathrm{Cl})\end{array}$ & $\mathrm{p}^{\mathrm{b}}$-trend \\
\hline Any na-NSAID (reference: no prescriptions) & $1.20(1.18-1.23)$ & $1.34(1.30-1.37)$ & $1.29(1.24-1.35)$ & $1.19(1.14-1.24)$ & 0.078 \\
Acetic acid derivatives & $1.20(1.17-1.22)$ & $1.33(1.29-1.38)$ & $1.27(1.20-1.36)$ & $1.19(1.09-1.30)$ & 0.002 \\
Propionic acid derivatives & $1.15(1.11-1.18)$ & $1.20(1.15-1.26)$ & $1.14(1.06-1.22)$ & $1.06(0.99-1.14)$ & 0.226 \\
Cox inhibitors & $1.20(1.12-1.29)$ & $1.20(1.09-1.32)$ & $1.24(1.04-1.47)$ & $0.94(0.76-1.15)$ & 0.128 \\
Systemic glucocorticoids & $1.12(1.08-1.17)$ & $1.17(1.11-1.23)$ & $1.24(1.15-1.32)$ & $1.08(1.01-1.16)$ & 0.697 \\
Inhaled glucocorticoids & $1.03(0.95-1.12)$ & $1.09(1.02-1.17)$ & $1.23(1.13-1.34)$ & $1.16(1.08-1.24)$ & 0.011 \\
Non-steroidal asthma medications & $1.06(0.99-1.14)$ & $1.06(0.99-1.14)$ & $1.17(1.09-1.26)$ & $1.12(1.07-1.18)$ & 0.098 \\
Immuno-suppressants & $0.58(0.38-0.89)$ & $0.75(0.60-0.92)$ & $1.02(0.86-1.21)$ & $1.06(0.96-1.17)$ & 0.001 \\
\hline
\end{tabular}

$C l$ confidence interval, $d d$ daily dose equivalents, NSAIDs non-steroidal anti-inflammatory drugs, OR odds ratio

${ }^{a}$ Cumulative daily dose equivalents of specific anti-inflammatory medications since July 2005, derived from the National Prescribed Drug Register based on anatomical therapeutic chemical (ATC) codes

${ }^{\mathrm{b}}$ Adjusted odds ratios derived from conditional logistic regression with adjustment for civil status, education level and Charlson comorbidity index. Analysis undertaken in the full dataset (2007-2012)

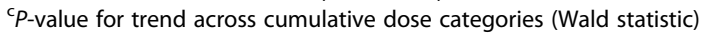


diabetic men which may reduce opportunities for diagnosis [34]. Though most of this evidence relates to type- 2 diabetes mellitus, a multinational study using diabetes registry data has reported lower risk of prostate cancer among men with T1DM [35]. While our findings also suggest decreased risk in men with T1DM, concerns remain about the accuracy of hospital records for type of diabetes, given the higher than expected prevalence of T1DM in our cohort.

With respect to allergies/asthma, we also observed higher risk for first hospitalisation within 12 months, which indicates detection bias may play a role. However, risk was also significantly elevated for first hospitalisation $>5$ years earlier suggesting the association may also involve pathways other than detection bias. Increased risk of prostate cancer has been reported previously among men with atopic diseases, specifically asthma [8], those using anti-asthma medications [21] and those positive for serum allergen-specific IgE, a marker of atopy [7]. However, the effect size in our study was more modest than other studies.

Counter to expectations, we observed a positive association between use of AIMs and risk of prostate cancer, with the strongest effects for non-aspirin-NSAIDs. Given the association was only observed for favourable risk prostate cancer and there was no clear dose response, we again suspect this finding reflects detection bias. While our findings are consistent with those of other large population-based studies (e.g. Skriver et al. 2016 [36], Denmark; Murad et al. 2011, UK [19]), they are inconsistent with meta-analyses which conclude that there is no association $[18,37]$ or a negative association [20] between prostate cancer and use of nonaspirin-NSAIDs. The meta-analysis by Lui et al. however, did indicate a positive association between non-aspirin-NSAIDs and prostate cancer risk in studies undertaken in Europe. Authors of the Danish population-based study also concluded that the positive association they observed was not likely to be causal [36]. However, findings from studies on the use of nonaspirin-NSAIDs and prostate cancer risk within the context of PSA screening trials do not support effects due to detection bias alone $[19,38]$.

With respect to inhaled glucocorticoids and prostate cancer risk we did find evidence of a dose-response effect, suggesting there may be a causal relationship. However, it is difficult to disentangle the effects of inhaled glucocorticoids from the effects of asthma, since most prescriptions would be for asthma. There are a number of plausible explanations for increased risk with frequent use of glucocorticoids. Firstly, immunosuppressive effects of glucocorticoids may weaken the body's defence against nascent or developing tumours [39]. Secondly, metabolic changes induced through sustained use of glucocorticoids (i.e. metabolic syndrome, obesity, hyperglycaemia) have been linked to increased cancer risk [40]. Thirdly, glucocorticoids may directly impact prostate cancer development through 'cross-talk' signalling of androgen receptors, leading to upregulation of androgen pathways which stimulate prostate cell growth [41]. However, the lack of dose response with systemic glucocorticoids, and only a weak association with unfavourable risk prostate cancer lessen the argument for a causal relationship. Even if the association between inhaled glucocorticoids and prostate cancer was causal, the effect of prolonged use of these drugs is very modest and would not outweigh the benefits in terms of controlling asthma.

We have used high-quality, registry-based databases to investigate the association between CIDs/AIMs and prostate cancer risk. In the context of autoimmune diseases, which are quite rare, having such a large study population and population-wide coverage is a major strength. Furthermore, we were able to investigate doseresponse relationships with respect to duration of CIDs and cumulative dose of AIMs. However, basing our exposure measure on hospital admission data will underestimate the true extent of CIDs since less severe inflammatory diseases (e.g. mild asthma, allergies) are often managed in community or outpatient settings. Likewise, prescription data may also be inaccurate since patients may not complete the course of medication prescribed. In addition we do not have information on prescription-free NSAID use. Furthermore, many AIMs have broad application beyond the management of inflammatory conditions (though this does not nullify their effect on prostate cancer). In terms of timing of initial diagnosis of CIDs, hospital admission data from 1998 may provide too short a time window to completely capture CID history, since conditions may have been diagnosed decades earlier. Even though we examined prostate cancer risk categories and trends according to exposure duration/dose, disentangling the effect of detection bias remains difficult without further information about primary health care visits and frequency of PSA testing.

\section{Conclusion}

This study found no consistent evidence linking prostate cancer risk to history of CIDs or use of AIMs. In general, the increased risk of prostate cancer is likely to be due to detection bias. However, findings suggesting possible associations with asthma/allergies and inhaled glucocorticoids warrant further investigation. More detailed clinical and translational research is required to better understand the relationship between chronic inflammation and prostate cancer risk, given limitations inherent in observational studies. 


\section{Additional files}

Additional file 1: Table S1. Adjusted Odds for association between AIMs exposure and PCa diagnosis in sensitivity analyses. Table S2. Adjusted Odds for association between AIMs exposure and PCa diagnosis in sensitivity analyses (DOCX $17 \mathrm{~kb}$ )

Additional file 2: List of conditions and medications included as exposures (DOCX $19 \mathrm{~kb}$ )

\section{Abbreviations}

AIM: Anti-Inflammatory Medications; ATC: Anatomical Therapeutic Chemical; CCl: Charlson Comorbidity Index; CID: Chronic Inflammatory Disease; ICD: International Classification of Diseases; Ig: Immunoglobulin; LISA: Swedish Longitudinal Integration Database for Health Insurance and Labour Market Studies; NPCR: National Prostate Cancer Register; NPR: National Patient Register; NSAID: Non Steriodal Anti-inflammatory Drug; OR: Odds Ratio; PCBaSe: Prostate Cancer Database Sweden; PSA: Prostate Serum Antigen; STD: Sexually Transmitted disease

\section{Acknowledgements}

This project was made possible by the continuous work of the National Prostate Cancer Register of Sweden steering group: Pär Stattin (Chairman), Camilla Thellenberg Karlsson, Ove Andrén, Ann-Sofin Fransson, Magnus Törnblom, Stefan Carlsson, Marie Hjälm-Eriksson, David Robinson, Mats Andén, Jonas Hugosson, Ingela Franck Lissbrant, Johan Stranne, Maria Nyberg, Göran Ahlgren, René Blom, Lars Egevad, Fredrik Sandin, and Karin Hellström. The authors also wish to acknowledge the expert advice provided by Sophia Karagiannis and Deborah Enting in relation to this study.

\section{Authors' contributions}

Study design and conception: MV, JA; acquisition of data: HG, PS; Statistical analysis of data: $K B, B R, H G$; interpretation of results: $K B, B R, D J, C H, L H, P S$ $M V$, JA; drafting manuscript KB: revision of manuscript: $K B, B R, D J, C H, L H, P S$ MV, JA. All authors approved the final manuscript.

\section{Funding}

Data collection and management within the National Prostate Cancer Register/ PCBaSe is supported by the Swedish Cancer Society (grant 2013/472). Dr. Beckmann is funded through a NHMRC Early Career Research Fellowship. The funding bodies had no input into the design, analysis and interpretation of this study, nor writing or approving this manuscript.

\section{Availability of data and materials}

The dataset analysed in this study was extracted from the Prostate Cancer Database Sweden (PCBaSe) and are available through the Professor Pär Stattin par.stattin@surgsci.uu.se on reasonable request.

\section{Ethics approval and consent to participate}

This study was approved by Research Ethics Board, Umeå University, Sweden. Waiver of informed consent has been granted for Register-based data.

\section{Consent for publication}

Not applicable.

\section{Competing interests}

The authors declare that they have no competing interests.

\section{Author details}

${ }^{1}$ UniSA Cancer Research Institute, University of South Australia, Adelaide, Australia. ${ }^{2}$ School of Cancer and Pharmaceutical Studies, Translational Oncology \& Urology Research (TOUR), King's College London, London, UK. ${ }^{3}$ Regional Cancer Centre Uppsala, Uppsala University Hospital, Uppsala, Sweden. ${ }^{4}$ Department of Biobank Research, Umea University, Umea, Sweden. ${ }^{5}$ Department of Surgical Sciences, Uppsala University Hospital, Uppsala, Sweden. ${ }^{6}$ Unit of Epidemiology, Institute of Environmental Medicine, Karolinska Institutet, Stockholm, Sweden. ${ }^{7}$ CLINTEC-department, Karolinska Institutet, Stockholm, Sweden.
Received: 18 December 2018 Accepted: 17 June 2019

Published online: 21 June 2019

\section{References}

1. Shacter E, Weitzman SA: Chronic inflammation and cancer. Oncology (Williston Park) 2002, 16(2):217-226, 229; discussion 230-212.

2. Sfanos KS, De Marzo AM. Prostate cancer and inflammation: the evidence. Histopathology. 2012;60(1):199-215

3. Vasto S, Carruba G, Candore G, Italiano E, Di Bona D, Caruso C. Inflammation and prostate cancer. Future Oncol. 2008;4(5):637-45.

4. Sutcliffe S, Platz EA. Inflammation in the etiology of prostate cancer: an epidemiologic perspective. Urol Oncol. 2007;25(3):242-9.

5. Sfanos KS, Hempel HA, De Marzo AM. The role of inflammation in prostate cancer. Adv Exp Med Biol. 2014;816:153-81.

6. Arthur R, Williams R, Garmo H, Holmberg L, Stattin P, Malmstrom H, Lambe M, Hammar N, Walldius G, Robinsson D, et al. Serum inflammatory markers in relation to prostate cancer severity and death in the Swedish AMORIS study. Int J Cancer. 2018;142(11):2254-62.

7. Wang $H$, Rothenbacher $D$, Low M, Stegmaier $C$, Brenner $H$, Diepgen TL. Atopic diseases, immunoglobulin $\mathrm{E}$ and risk of cancer of the prostate, breast, lung and colorectum. Int J Cancer. 2006;119(3):695-701.

8. Talbot-Smith A, Fritschi L, Divitini ML, Mallon DF, Knuiman MW. Allergy, atopy, and cancer: a prospective study of the 1981 Busselton cohort. Am J Epidemiol. 2003;157(7):606-12.

9. Hemminki K, Li X, Sundquist J, Sundquist K. Cancer risks in ulcerative colitis patients. Int J Cancer. 2008;123(6):1417-21.

10. Hemminki K, Li X, Sundquist J, Sundquist K. Cancer risks in Crohn disease patients. Ann Oncol. 2009;20(3):574-80.

11. Ji J, Liu X, Sundquist K, Sundquist J, Hemminki K. Cancer risk in patients hospitalized with polymyalgia rheumatica and giant cell arteritis: a followup study in Sweden. Rheumatology (Oxford). 2010;49(6):1158-63.

12. Klaassen Z, Tatem A, Augusta G, Patil S, Livingston N, Madi R, Terris M, Moses K. Prostate cancer incidence in patients with autoimmune diseases: a populationbased analysis (conference abstract). J Urol. 2013;189(4S (Suppl):e135.

13. Gonzalez-Perez A, Fernandez-Vidaurre C, Rueda A, Rivero E, Garcia Rodriguez LA. Cancer incidence in a general population of asthma patients. Pharmacoepidemiol Drug Saf. 2006;15(2):131-8.

14. Platz EA, Drake CG, Wilson KM, Sutcliffe S, Kenfield SA, Mucci LA, Stampfer MJ, Willett WC, Camargo CA Jr, Giovannucci E. Asthma and risk of lethal prostate cancer in the health professionals follow-up study. Int J Cancer. 2015:137(4):949-58.

15. Vena JE, Bona JR, Byers TE, Middleton E Jr, Swanson MK, Graham S. Allergy-related diseases and cancer: an inverse association. Am J Epidemiol. 1985;122(1):66-74.

16. Bernatsky S, Ramsey-Goldman R, Gordon C, Clarke AE. Prostate cancer in systemic lupus erythematosus. Int J Cancer. 2011;129(12):2966-9.

17. Parikh-Patel A, White $\mathrm{RH}$, Allen M, Cress R. Risk of cancer among rheumatoid arthritis patients in California. Cancer Causes Control. 2009;20(6):1001-10.

18. Liu Y, Chen JQ, Xie L, Wang J, Li T, He Y, Gao Y, Qin X, Li S. Effect of aspirin and other non-steroidal anti-inflammatory drugs on prostate cancer incidence and mortality: a systematic review and meta-analysis. BMC Med. 2014;12:55.

19. Murad AS, Down L, Davey Smith G, Donovan JL, Athene Lane J, Hamdy FC, Neal DE, Martin RM. Associations of aspirin, nonsteroidal anti-inflammatory drug and paracetamol use with PSA-detected prostate cancer: findings from a large, population-based, case-control study (the ProtecT study). Int J Cancer. 2011;128(6):1442-8.

20. Wang $X$, Lin YW, Wu J, Zhu Y, Xu XL, Xu X, Liang Z, Hu ZH, Li SQ, Zheng XY, et al. Meta-analysis of nonsteroidal anti-inflammatory drug intake and prostate cancer risk. World J Surg Oncol. 2014;12:304.

21. Severi G, Baglietto L, Muller DC, English DR, Jenkins MA, Abramson MJ, Douglass JA, Hopper JL, Giles GG. Asthma, asthma medications, and prostate cancer risk. Cancer Epidemiol Biomark Prev. 2010;19(9):2318-24.

22. Dennis LK, Lynch CF, Torner JC. Epidemiologic association between prostatitis and prostate cancer. Urology. 2002;60(1):78-83.

23. Van Hemelrijck M, Wigertz A, Sandin F, Garmo H, Hellstrom K, Fransson P, Widmark A, Lambe M, Adolfsson J, Varenhorst E, et al. Cohort profile: the National Prostate Cancer Register of Sweden and prostate Cancer data base Sweden 2.0. Int J Epidemiol. 2013;42(4):956-67. 
24. Charlson ME, Pompei P, Ales KL, Mackenzie CR. A new method of classifying prognostic comorbidity in longitudinal studies: development and validation. J Chronic Dis. 1987;40(5):373-83.

25. National working group for cancer care: National Prostate Care Program. In.: Regional Cancer Centre Uppsala, Orebro; 2015.

26. Nordstrom T, Aly M, Clements MS, Weibull CE, Adolfsson J, Gronberg $H$. Prostate-specific antigen (PSA) testing is prevalent and increasing in Stockholm County, Sweden, despite no recommendations for PSA screening: results from a population-based study, 2003-2011. Eur Urol. 2013;63(3):419-25.

27. Bratt O, Berglund A, Adolfsson J, Johansson JE, Tornblom M, Stattin P, Steering Committee of the Swedish National Prostate Cancer R. Prostate cancer diagnosed after prostate-specific antigen testing of men without clinical signs of the disease: a population-based study from the National Prostate Cancer Register of Sweden. Scand J Urol Nephrol. 2010;44(6):384-90.

28. Hayat Roshanai A, Nordin K, Berglund G. Factors influencing primary care physicians' decision to order prostate-specific antigen (PSA) test for men without prostate cancer. Acta Oncol. 2013;52(8):1602-8.

29. Pucheril D, Dalela D, Sammon J, Sood A, Sun M, Trinh QD, Menon M, Abdollah $\mathrm{F}$. The influence of physician recommendation on prostatespecific antigen screening. Urol Oncol. 2015;33(10):424 e421-7.

30. Kotwal AA, Schumm P, Mohile SG, Dale W. The influence of stress, depression, and anxiety on PSA screening rates in a nationally representative sample. Med Care. 2012;50(12):1037-44.

31. Bonovas S, Filioussi K, Tsantes A. Diabetes mellitus and risk of prostate cancer: a meta-analysis. Diabetologia. 2004;47(6):1071-8.

32. Fall K, Garmo H, Gudbjornsdottir S, Stattin P, Zethelius B. Diabetes mellitus and prostate cancer risk; a nationwide case-control study within PCBaSe Sweden. Cancer Epidemiol Biomark Prev. 2013;22(6):1102-9.

33. Kasper JS, Giovannucci E. A meta-analysis of diabetes mellitus and the risk of prostate cancer. Cancer Epidemiol Biomark Prev. 2006;15(11):2056-62.

34. Pierce BL. Why are diabetics at reduced risk for prostate cancer? A review of the epidemiologic evidence. Urol Oncol. 2012;30(5):735-43.

35. Carstensen B, Read SH, Friis S, Sund R, Keskimaki I, Svensson AM, Ljung R, Wild SH, Kerssens $\mathrm{J}$, Harding JL, et al. Cancer incidence in persons with type 1 diabetes: a five-country study of 9,000 cancers in type 1 diabetic individuals. Diabetologia. 2016;59(5):980-8.

36. Skriver C, Dehlendorff C, Borre M, Brasso K, Sorensen HT, Hallas J, Larsen SB, Tjonneland A, Friis S. Low-dose aspirin or other nonsteroidal antiinflammatory drug use and prostate cancer risk: a nationwide study. Cancer Causes Control. 2016;27(9):1067-79.

37. Mahmud SM, Franco EL, Aprikian AG. Use of nonsteroidal anti-inflammatory drugs and prostate cancer risk: a meta-analysis. Int J Cancer. 2010;127(7):1680-91.

38. Veitonmaki T, Murtola TJ, Maattanen L, Taari K, Stenman UH, Tammela TL, Auvinen A. Prostate cancer risk and nonsteroidal antiinflammatory drug use in the Finnish prostate cancer screening trial. Br J Cancer. 2014;111(7):1421-31.

39. Oehling AG, Akdis CA, Schapowal A, Blaser K, Schmitz M, Simon HU. Suppression of the immune system by oral glucocorticoid therapy in bronchial asthma. Allergy. 1997;52(2):144-54.

40. Fardet L, Feve B. Systemic glucocorticoid therapy: a review of its metabolic and cardiovascular adverse events. Drugs. 2014;74(15):1731-45.

41. Narayanan S, Srinivas S, Feldman D. Androgen-glucocorticoid interactions in the era of novel prostate cancer therapy. Nat Rev Urol. 2016;13(1):47-60.

\section{Publisher's Note}

Springer Nature remains neutral with regard to jurisdictional claims in published maps and institutional affiliations.

Ready to submit your research? Choose BMC and benefit from:
- fast, convenient online submission
- thorough peer review by experienced researchers in your field
- rapid publication on acceptance
- support for research data, including large and complex data types
- gold Open Access which fosters wider collaboration and increased citations
- maximum visibility for your research: over 100M website views per year
At BMC, research is always in progress.
Learn more biomedcentral.com/submissions

